\title{
HIGHLIGHTS
}

HYPERTENSION

\section{Hypertension development in normotensive adolescents}

Increasing levels of blood pressure in adolescents-even levels within the low-normotensive range-are linearly associated with an increasing risk of incident hypertension in later life, according to a report in Hypertension.

Previous studies had shown that prehypertension in adolescence is associated with an increased risk of hypertension in early adulthood, but large-scale studies investigating the development of hypertension in normotensive adolescents were lacking.

Amir Tirosh and co-workers followed 26,980 adolescents $(23,191$ of whom were male) who were in the Medical Corps of the Israeli army and were part of the Metabolic Lifestyle and Nutrition Assessment in Young Adults (MELANY) cohort. Individuals included in Tirosh et al.'s analysis had a blood pressure $<140 / 90 \mathrm{mmHg}$ at baseline (that is, at their initial medical evaluation before recruitment for military service; mean age 17.4 years). Blood pressure and BMI measurements were recorded every $3-5$ years between the ages of 25 years and 42 years.

The researchers found that male individuals had a lower mean baseline BMI but higher mean baseline systolic and diastolic blood pressures than female individuals. During follow-up (up to the age of 42 years), 3,810 individuals developed hypertension (defined as blood pressure $\geq 140 / 90 \mathrm{mmHg}$ ). A Cox regression survival analysis adjusted for age, sex and baseline blood pressure levels revealed that men were 3-4 times more likely than women to develop hypertension during follow-up. The cumulative risk of hypertension development increased gradually with increasing levels of blood pressure during adolescence (that is, at baseline), in both male and female participants. In addition, the risk of developing hypertension in the future increased with increasing adolescent

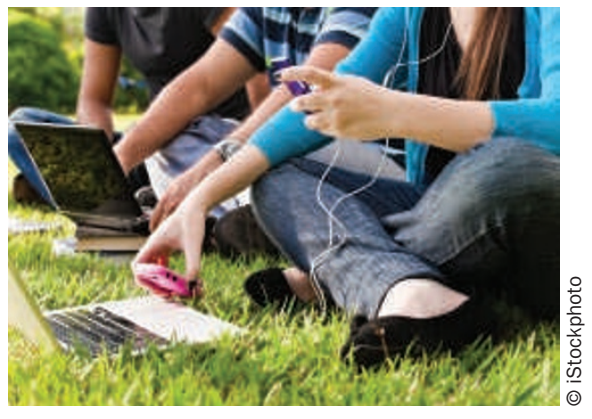

BMI categories (underweight, normal weight, overweight and obese) in men. In women, however, the relationship between increasing BMI during adolescence and hypertension in later life was only significant in individuals classified as overweight or obese at baseline.

"Taken together, these very simple measurements of BMI and blood pressure may provide a simple and useful tool that can serve as a 'red flag' to detect subgroups of teenagers at high risk of developing hypertension early in life," notes Tirosh.

\section{Rebecca Ireland}

Original article Tirosh, A. et al. Progression of normotensive adolescents to hypertensive adults: a study of 26980 teenagers. Hypertension 56, 203-209 (2010) 